University of South Carolina

Scholar Commons

$5-13-2002$

\title{
Crack-Free Thick AlGaN Grown on Sapphire using AIN/AIGaN Superlattices for Strain Management
}

\author{
J. P. Zhang \\ H. M. Wang \\ M. E. Gaevski \\ C. Q. Chen \\ Q. Fareed
}

See next page for additional authors

Follow this and additional works at: https://scholarcommons.sc.edu/elct_facpub

Part of the Electromagnetics and Photonics Commons, and the Other Electrical and Computer Engineering Commons

Publication Info

Published in Applied Physics Letters, Volume 80, Issue 19, 2002, pages 3542-3544.

(C)Applied Physics Letters 2002, American Institute of Physics (AIP).

Zhang, J. P., Wang, H. M., Gaevski, M. E., Chen, C. Q., Fareed, Q., Yang, J. W., Simin, G., \& Khan, M. A. (13

May 2002). Crack-Free Thick AIGaN Grown on Sapphire using AIN/AIGaN Superlattices for Strain

Management. Applied Physics Letters, 80 (19), 3542-3544. http://dx.doi.org/10.1063/1.1477620

This Article is brought to you by the Electrical Engineering, Department of at Scholar Commons. It has been accepted for inclusion in Faculty Publications by an authorized administrator of Scholar Commons. For more information, please contact digres@mailbox.sc.edu. 


\section{Author(s)}

J. P. Zhang, H. M. Wang, M. E. Gaevski, C. Q. Chen, Q. Fareed, J. W. Yang, Grigory Simin, and M. Asif Khan 


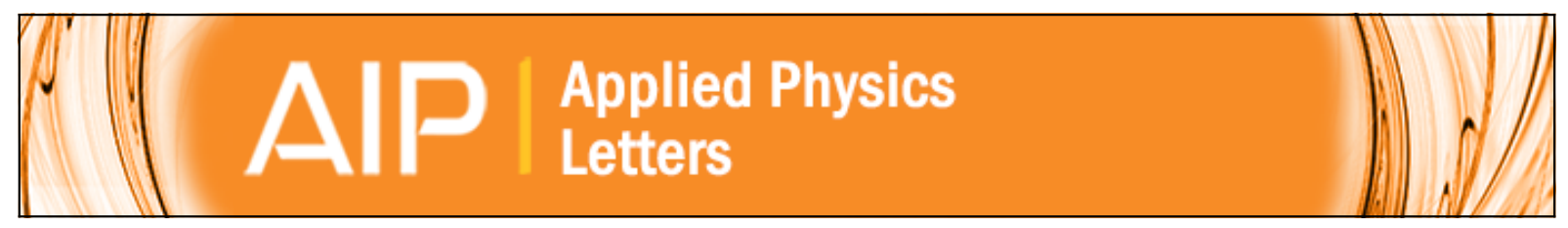

Crack-free thick AIGaN grown on sapphire using AIN/AIGaN superlattices for strain management

J. P. Zhang, H. M. Wang, M. E. Gaevski, C. Q. Chen, Q. Fareed, J. W. Yang, G. Simin, and M. Asif Khan

Citation: Applied Physics Letters 80, 3542 (2002); doi: 10.1063/1.1477620

View online: http://dx.doi.org/10.1063/1.1477620

View Table of Contents: http://scitation.aip.org/content/aip/journal/apl/80/19?ver=pdfcov

Published by the AIP Publishing

\section{Articles you may be interested in}

Selective lateral electrochemical etching of a GaN-based superlattice layer for thin film device application Appl. Phys. Lett. 102, 152112 (2013); 10.1063/1.4802274

Reduction of threading dislocations in crack-free $\mathrm{AIGaN}$ by using multiple thin $\mathrm{Si} x \mathrm{Al} 1-\mathrm{x} \mathrm{N}$ interlayers Appl. Phys. Lett. 83, 4140 (2003); 10.1063/1.1628397

Electrical properties of strained AIN/GaN superlattices on GaN grown by metalorganic vapor phase epitaxy Appl. Phys. Lett. 80, 802 (2002); 10.1063/1.1446204

Metalorganic vapor phase epitaxy growth of crack-free AIN on GaN and its application to high-mobility AIN/GaN superlattices

Appl. Phys. Lett. 79, 3062 (2001); 10.1063/1.1416169

Carbon delta-doped AIGaAs grown by metalorganic vapor phase epitaxy

J. Appl. Phys. 90, 1660 (2001); 10.1063/1.1382826

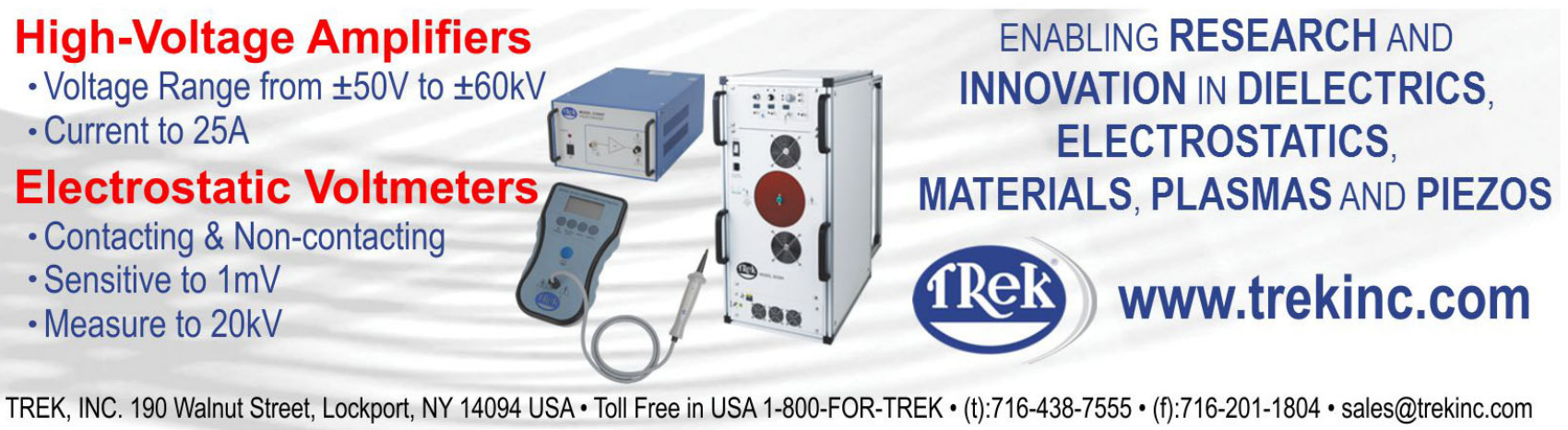




\title{
Crack-free thick AIGaN grown on sapphire using AIN/AIGaN superlattices for strain management
}

\author{
J. P. Zhang, H. M. Wang, M. E. Gaevski, C. Q. Chen, Q. Fareed, J. W. Yang, G. Simin, \\ and M. Asif Khan ${ }^{\text {a) }}$ \\ Department of Electrical Engineering, University of South Carolina, Columbia, South Carolina 29208
}

(Received 1 December 2001; accepted for publication 21 March 2002)

\begin{abstract}
We report on an AlN/AlGaN superlattice approach to grow high-Al-content thick $n^{+}$-AlGaN layers over $c$-plane sapphire substrates. Insertion of a set of AlN/AlGaN superlattices is shown to significantly reduce the biaxial tensile strain, thereby resulting in 3- $\mu$ m-thick, crack-free $\mathrm{Al}_{0.2} \mathrm{Ga}_{0.8} \mathrm{~N}$ layers. These high-quality, low-sheet-resistive layers are of key importance to avoid current crowding in quaternary AlInGaN multiple-quantum-well deep-ultraviolet light-emitting diodes over sapphire substrates. (C) 2002 American Institute of Physics. [DOI: 10.1063/1.1477620]
\end{abstract}

Deep-ultraviolet (UV) light-emitting diodes (LEDs) and laser diodes are key to applications such as solid-state whitelight, biochemical detection and lithography. Recently, several groups have reported on LEDs with emission wavelengths from 340 to $360 \mathrm{~nm}$ using AlGaN layers in the active region. ${ }^{1-3}$ Alternatively, we have reported on deep-UV LEDs on sapphire with emission wavelengths from 305 to $340 \mathrm{~nm}$ with a quaternary AlInGaN multiple-quantum-well (MQW) active region. ${ }^{4-6}$ We used a unique pulsed atomic-layer epitaxy procedure to deposit the AlInGaN MQWs for the active region. ${ }^{6,7}$ In our design the LED structure was grown over the sapphire substrate. This choice, compared to the $\mathrm{SiC}$ substrate, allows for much higher light extraction efficiency, which is of crucial importance for deep-UV devices. The total emitted powers and the differential quantum efficiencies were found to decrease with decreasing emission wavelength. ${ }^{5}$ Some of the key reasons for this performance degradation with wavelength were the thickness, doping level, and the quality of the high-Al-content $n^{+}$-AlGaN cladding layers, ${ }^{8,9}$ which are responsible for the quality of the above-lying MQW layers as well as for the lateral current spreading in the device. The use of a thin AlGaN cladding layer combined with lateral device geometries led to a severe current crowding. ${ }^{10}$ To circumvent these problems, crackfree, thick $(\geqslant 3 \mu \mathrm{m})$ AlGaN layers are required. ${ }^{10}$ Several groups reported on different approaches to grow thick AlGaN layers over GaN buffers. ${ }^{11,12}$ However, the use of GaN layers drastically decreases the light extraction efficiency in deep-UV emitters due to strong absorption. We now report an approach of using a set of AlN/AlGaN superlattices (SLs) to reduce the biaxial tensile strain and successfully grow $3.0-\mu \mathrm{m}$-thick $\mathrm{Al}_{0.2} \mathrm{Ga}_{0.8} \mathrm{~N}$ on sapphire without any cracks. Scanning electron microscopy (SEM), photoluminescence (PL), x-ray diffraction (XRD), and transmission spectrum were used to investigate the effect of the SL strain engineering on the quality of the resulting thick $\mathrm{AlGaN}$ layer. While this letter was under preparation, Feltin et al. reported

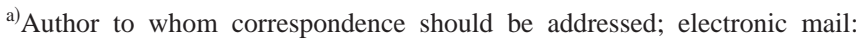
asif@engr.sc.edu
}

a similar AlN/GaN superlattice insertion approach to control cracking in thick $\mathrm{GaN}$ layers grown over $\mathrm{Si}(111)$ substrates. ${ }^{13}$

The AlGaN layers for this study were deposited on basal plane sapphire substrates using low-pressure metalorganic chemical-vapor deposition. Trimethyl aluminum, trmethylgallium, and $\mathrm{NH}_{3}$ were used as the precursors. Three sets of $\mathrm{Al}_{0.2} \mathrm{Ga}_{0.8} \mathrm{~N}$ samples were deposited, differing only in the buffer layer configuration. Samples in set A had a $250-\AA$ thick low-temperature (LT) AlN buffer prior to the $\mathrm{Al}_{0.2} \mathrm{Ga}_{0.8} \mathrm{~N}$ layers and are referred to as LT samples. Samples in set B have an additional high-temperature (HT) grown 40-nm-thick AlN intermediate layer in between the LT-AlN buffer and the $\mathrm{Al}_{0.2} \mathrm{Ga}_{0.8} \mathrm{~N}$ epilayers. We will refer this set B as the HT samples. Samples in set C are referred to as the SL samples. For set C, first a $250 \AA$ LT-AlN buffer was deposited on sapphire, followed by a $0.2-\mu \mathrm{m}$-thick HTgrown $\mathrm{Al}_{0.2} \mathrm{Ga}_{0.8} \mathrm{~N}$, and then a ten-period $\mathrm{AlN} / \mathrm{Al}_{0.2} \mathrm{Ga}_{0.8} \mathrm{~N}$ SL. Finally, $\mathrm{Al}_{0.2} \mathrm{Ga}_{0.8} \mathrm{~N}$ layers with thickness ranging from 1 to $3 \mu \mathrm{m}$ were grown. For all these samples, the LT buffers and HT epilayers' growth temperatures were 600 and $1060^{\circ} \mathrm{C}$, respectively. The growth pressure was 40 Torr and the V/III ratio 550. For the $n$-type doping study, disilane was used as the dopant.

Figure 1 shows the SEM images of the samples under investigation. For the LT and HT samples, we always observe cracks when the top $\mathrm{Al}_{0.2} \mathrm{Ga}_{0.8} \mathrm{~N}$ exceeds $1.2 \mu \mathrm{m}$ [Figs. 1(a) and 1(b)]. However, the HT sample showed some reduction in the crack density. For the SL sample, no cracks were observed for the $\mathrm{Al}_{0.2} \mathrm{Ga}_{0.8} \mathrm{~N}$ layer even as thick as $3.0 \mu \mathrm{m}$ [Fig. 1(c)]. Shown in Fig. 1(d) is the SEM cross-section view of the SL sample, where the strain-engineering SL structure is clearly seen. From the enlarged view we find the total thickness of the SL region to be around $320 \mathrm{~nm}$, implying a SL period of $32 \mathrm{~nm}$. We also used cathodoluminescence mappings to estimate the material uniformity. The result indicates that the SL sample is more homogeneous than the LT and HT samples.

Figure 2 shows the room-temperature (RT) PL and transmission spectra of the 3- $\mu$ m-thick SL sample. The PL spectra were measured at RT using a pulsed excimer laser $(\lambda$ $=193 \mathrm{~nm}, \tau=8 \mathrm{~ns}$ ), with an average excitation power den- 

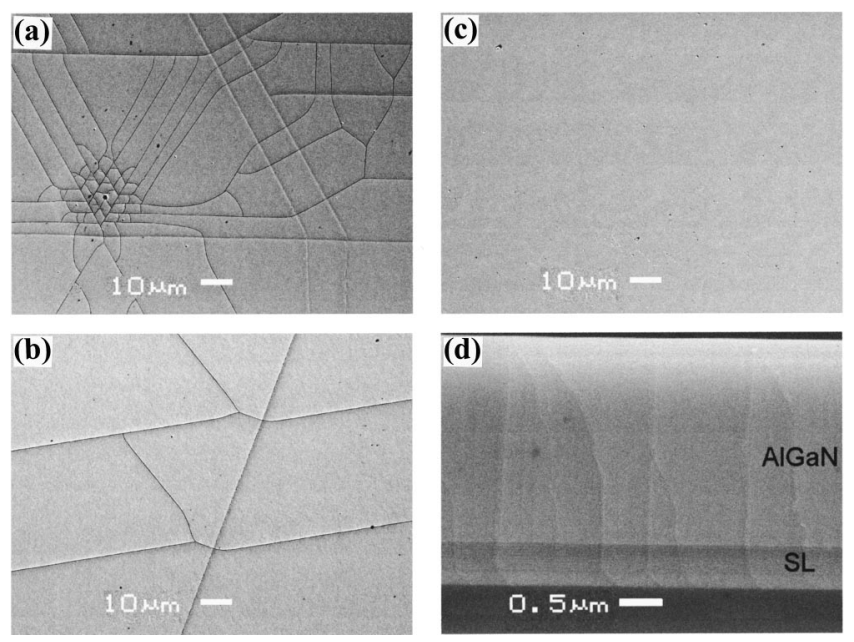

FIG. 1. Plane-view SEM images (backscattering mode) of the LT sample (a), HT sample (b), and the $3.0 \mu \mathrm{m}$ SL sample (c). Cross-section view of the SL sample (d).

sity of $0.2 \mathrm{MW} / \mathrm{cm}^{2}$. As seen, the very small Stokes shift between the PL and transmission spectra indicates the high quality of the SL sample. This is also confirmed by the small RT PL linewidth $(6 \mathrm{~nm})$. In the inset to Fig. 2 we compare the RT PL of the LT, HT, and SL samples of different thicknesses with the same Al mole fraction. As seen, the PL spectra of the LT and HT samples show a significant redshift as compared to the SL samples, implying a biaxial tensile strain existing in the LT and HT samples. This can explain the observed cracking in LT and HT samples as their thickness exceeds about $1 \mu \mathrm{m}$. Also, we can see that the PL in the SL samples shows a slight redshift with increasing thickness. This shift we believe is due to strain modification as the top $\mathrm{AlGaN}$ gets thick. The redshift, however, saturates when the total thickness is over $2.2 \mu \mathrm{m}$. We note that the PL intensity of the SL samples increases with thickness. Since the PL spectra were measured using excimer laser excitation with extremely high absorption coefficient, this intensity increase indicates the material quality improvement.

XRD rocking-curve measurements indicate that the structural quality of the SL samples are much better than that of the LT and HT samples. The full width at half maximum

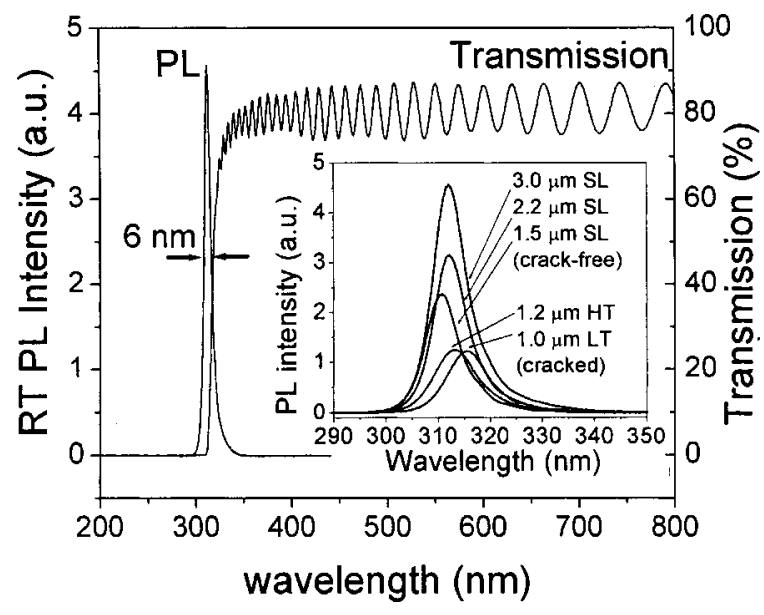

FIG. 2. Transmission and PL spectra of the $3.0 \mu \mathrm{m}$ SL sample. The inset shows RT PL spectra of the LT, HT, and SL samples with different thicknesses of the top $\mathrm{Al}_{0.2} \mathrm{Ga}_{0.8} \mathrm{~N}$ layer.

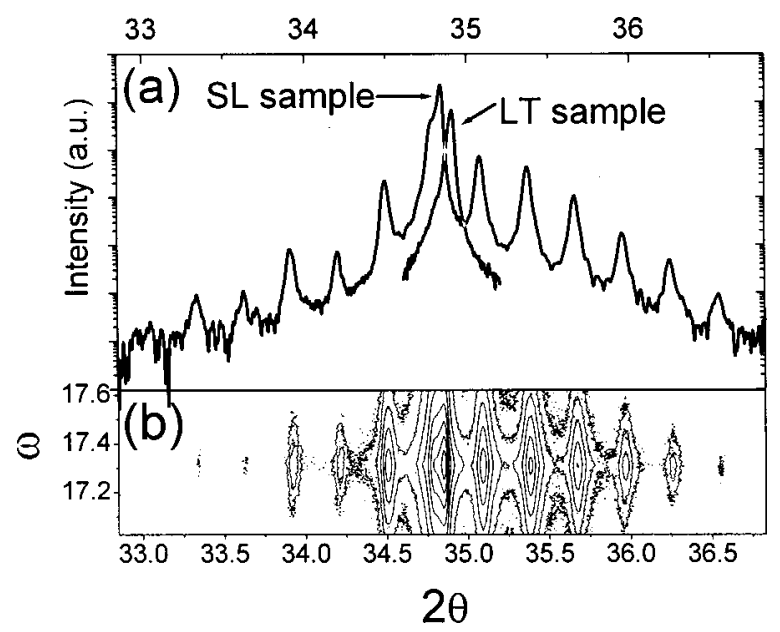

FIG. 3. (0002) $2 \theta-\omega$ scans of the $3.0 \mu \mathrm{m}$ SL and LT sample (a); (0002) mapping of the $3.0 \mu \mathrm{m}$ SL sample (b).

values of the (0002) $\omega$ scans for $1.5 \mu \mathrm{m} \mathrm{SL}$ and LT/HT samples are 6 and 16 arcmin, respectively. For the asymmetrical (20-24) $\omega$ scans, these values are, respectively, 11.5 and 38 arcmin. Figure 3(a) shows the (0002) $2 \theta-\omega$ scans of the $3.0 \mu \mathrm{m} \mathrm{SL}$ and the LT samples. As seen, for the SL sample, up to 12 satellite peaks are clearly resolved. The strongest peak in Fig. 3(a) is attributed to the top thick $\mathrm{Al}_{0.2} \mathrm{Ga}_{0.8} \mathrm{~N}$ epilayers; its low-angle shoulder is the superlattice zeroth-order peak, reflecting the SL average composition and overall strain. The average $\mathrm{Al}$ mole fraction $(\sim 40 \%)$ in the $\mathrm{AlN} / \mathrm{Al}_{0.2} \mathrm{Ga}_{0.8} \mathrm{~N}$ SL is larger than that in the top AlGaN epilayer (20\%), therefore, the SL zeroth-order peak appearing to the lower-angle position indicates that the SL structure is under a stronger biaxial compression as compared to the top AlGaN layer. This will be confirmed in the following analysis. From the angular distance between the neighboring satellite peaks, the average period of the SL is again confirmed to be $32 \mathrm{~nm}$. Moreover, for the same $\mathrm{Al}$ fraction, the LT sample is under more biaxial tensile strain than the SL sample, as inferred from its higher angular peak position. Thus, these XRD data confirm the PL data in the inset of Fig. 2. Our data clearly show that the effect of insertion of the AlN/AlGaN SL is to reduce the biaxial tensile strain of the top AlGaN layer. Figure 3(b) shows the symmetrical mapping around the (0002) reflection for the SL sample used in Fig. 3(a). As seen, the SL satellite peaks lie in the same line direction relative to the top $\mathrm{Al}_{0.2} \mathrm{Ga}_{0.8} \mathrm{~N}$ surface normal, confirming that the top $\mathrm{Al}_{0.2} \mathrm{Ga}_{0.8} \mathrm{~N}$ had grown on axis to the AlN/AlGaN SL strain-engineering layer.

More-detailed strain status in the SL sample can be extracted from the asymmetrical XRD mapping. In Fig. 4 we show the (20-24) asymmetrical XRD mapping for the 3.0 $\mu \mathrm{m}$ SL sample. The top thick $\mathrm{Al}_{0.2} \mathrm{Ga}_{0.8} \mathrm{~N}$ reciprocal lattice point is not in vertical $Q_{x}$ alignment with the SL satellites, indicating that it was not pseudomorphically grown on the strain-engineering SL, it is partly relaxed. Since the SL zeroth-order peak merges into the high-angle tail of the top $\mathrm{Al}_{0.2} \mathrm{Ga}_{0.8} \mathrm{~N}$ peak, to estimate the lattice constants, we take the angle in the middle of the \pm 1 satellite peaks as the zeroth-peak position. Therefore, from Figs. 3 and 4, for the top $\mathrm{Al}_{0.2} \mathrm{Ga}_{0.8} \mathrm{~N}$ epilayer of the SL sample, $a=3.1590 \AA$ and $c=5.1461 \AA$; for the SL strain-engineering structure, the av- 


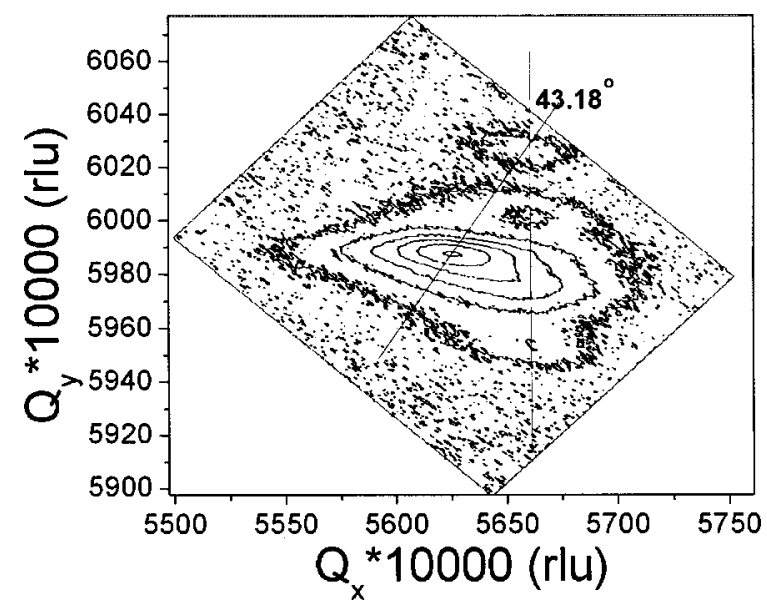

FIG. 4. (20-24) mapping of the $3.0 \mu \mathrm{m}$ SL sample in reciprocal space.

erage lattice constants are $a=3.1482 \AA$ and $c=5.1518 \AA$; and for the $\mathrm{Al}_{0.2} \mathrm{Ga}_{0.8} \mathrm{~N}$ of the LT sample, $c=5.1362 \AA$. According to Ref. 14, for free-standing $\mathrm{Al}_{0.2} \mathrm{Ga}_{0.8} \mathrm{~N}, \quad a$ $=3.1736 \AA$ and $c=5.1382 \AA$. We can see that although most of the strain was relaxed by the cracks, the LT sample is still under biaxial tensile strain, while the SL sample is overall under biaxial compressive strain. The SL itself experiences a higher biaxial compression than the top $\mathrm{Al}_{0.2} \mathrm{Ga}_{0.8} \mathrm{~N}$ epilayer. Thus, biaxial compression was partly transferred from the strain-engineering SL to the top $\mathrm{Al}_{0.2} \mathrm{Ga}_{0.8} \mathrm{~N}$ epilayer.

The cracks are caused by biaxial tensile strain. Usually, strain can be released by the creation of misfit dislocations. In III nitrides, because of the absence of an effective slip system, ${ }^{15}$ dislocations are difficult to generate and glide. On the other hand, the development of biaxial tensile strain in AlGaN grown on $c$-face sapphire is not well understood. From the thermal expansion coefficient and lattice constant mismatch, GaN grown on $c$-face sapphire will be under biaxial compression, as being observed in most of the experiments. However, experimentally, the strain status of AlGaN over sapphire is quite complicated. Krost et al. ${ }^{16}$ observed that $\mathrm{Al}_{x} \mathrm{Ga}_{1-x} \mathrm{~N}(x<0.4)$ grown on sapphire tends to settle the in-plane lattice constant according to $a_{\mathrm{GaN}}: a_{\text {sapphire }}$ $=2: 3$. Therefore, for $\mathrm{Al}$ fraction $x<0.18$, the $\mathrm{Al}_{x} \mathrm{Ga}_{1-x} \mathrm{~N}$ on $c$-face sapphire is under biaxial tension; for $0.18<x<0.25$, $\mathrm{Al}_{x} \mathrm{Ga}_{1-x} \mathrm{~N}$ is nearly strain free; for $0.25<x<0.4$, $\mathrm{Al}_{x} \mathrm{Ga}_{1-x} \mathrm{~N}$ is under biaxial compression. Our observations do not fully agree with this. The LT/HT samples used in this study all have an $\mathrm{Al}$ fraction of $20 \%$, but they do not show the strain-free condition. On the contrary, they are under strong biaxial tension. For the strain-engineering SL, the average $\mathrm{Al}$ fraction is close to $40 \%$. From our XRD data this SL structure is under biaxial compressive strain, which is in accordance with the results of Ref. 16. Therefore, the SL structure transfers the biaxial compressive strain to the top $\mathrm{Al}_{0.2} \mathrm{Ga}_{0.8} \mathrm{~N}$ epilayer, preventing it from cracking.
Finally, the electrical properties of the Si-doped SL sample were measured. From on-wafer Hall measurements, a RT mobility of $130 \mathrm{~cm}^{2} / \mathrm{V} \mathrm{s}$ was obtained for a doping level of $2.5 \times 10^{18} \mathrm{~cm}^{-3}$. These values are very close to those measured for $n$-doped GaN layers on sapphire. ${ }^{17}$ In contrast, the RT mobility for the LT/HT samples was around 30-60 $\mathrm{cm}^{2} / \mathrm{V}$ s. This clearly establishes a superior electrical quality for the $\mathrm{AlGaN}$ layer resulting from the SL insertion procedure. We also note that based on the SL samples, recently we demonstrated milliwatt deep-UV LEDs operating at $326 \mathrm{~nm}$ ( $2 \mathrm{~mW}$ at $160 \mathrm{~mA}$ pulsed current). These LEDs showed stable operation and a negligible current crowding effect.

In conclusion, we studied the effect of AlN/AlGaN SLs on the growth of thick AlGaN layers on sapphire. With the conventional LT-AlN buffer AlGaN grown on sapphire suffers from strong biaxial tension, which inevitably causes cracks as the thickness increases. An insertion of the AlN/ $\mathrm{AlGaN}$ SL structure reduces the tensile strain, and can even convert it from tensile to compressive. XRD mapping data were used to analyze the strain modified by the SL strainengineering layer. This strain management allowed us to grow a 3 - $\mu$ m-thick, crack-free $\mathrm{Al}_{0.2} \mathrm{Ga}_{0.8} \mathrm{~N}$ layer over sapphire substrates. The use of these thick $n^{+}$-AlGaN layers as buffer layers for deep-UV LEDs can significantly reduce current crowding and improve the quality of the active MQW region.

${ }^{1}$ T. Nishida, H. Saito, and N. Kobayashi, Appl. Phys. Lett. 78, 3927 (2001).

${ }^{2}$ A. Kinoshita, H. Hirayama, M. Ainoya, Y. Aoyagi, and A. Hirata, Appl. Phys. Lett. 77, 175 (2000).

${ }^{3}$ N. Otsuka, A. Tsujimura, Y. Hasegawa, G. Sugahara, M. Kume, and Y. Ban, Jpn. J. Appl. Phys., Part 2 39, L445 (2000).

${ }^{4}$ V. Adivarahan, A. Chitnis, J. P. Zhang, M. Shatalov, J. W. Yang, G. Simin, M. Asif Khan, M. Shur, and R. Gaska, Appl. Phys. Lett. 79, 4240 (2001).

${ }^{5}$ M. Asif Khan, V. Adivarahan, J. P. Zhang, C. Chen, E. Kuokstis, A. Chitnis, M. Shatalov, J. W. Yang, and G. Simin, Jpn. J. Appl. Phys., Part 2 40, L1308 (2001).

${ }^{6}$ J. P. Zhang, V. Adivarahan, H. M. Wang, Q. Fareed, E. Koukstis, A. Chitnis, M. Shatalov, J. W. Yang, G. Simin, M. Asif Khan, M. Shur, and R. Gaska, Jpn. J. Appl. Phys., Part 2 40, L921 (2001).

${ }^{7}$ J. P. Zhang, E. Kuokstis, Q. Fareed, H. M. Wang, J. Yang, G. Simin, M. Asif Khan, R. Gaska, and M. Shur, Appl. Phys. Lett. 79, 925 (2001).

${ }^{8}$ H. Amano and I. Akasaki, Opt. Mater. 19, 219 (2001).

${ }^{9}$ C.-R. Lee, S.-J. Son, K.-W. Seol, J.-M. Yeo, H.-K. Ahn, and Y.-J. Park, J. Cryst. Growth 226, 215 (2001).

${ }^{10}$ M. Shatalov, J. P. Zhang, A. Chitnis, V. Adivarahan, J. W. Yang, G. Simin, and M. Asif Khan, IEEE J. Sel. Top. Quantum Electron. 8 (2002).

${ }^{11}$ S. Kamiyama, M. Iwaya, N. Hayashi, T. Takeuchi, H. Amano, I. Akasaki, S. Watanabe, Y. Kaneko, and N. Yamada, J. Cryst. Growth 223, 83 (2001).

${ }^{12}$ H. Amano, M. Iwaya, N. Hayashi, T. Kashima, S. Nitta, C. Wetzel, and I. Akasaki, Phys. Status Solidi B 216, 683 (1999).

${ }^{13}$ E. Feltin, B. Beaumont, M. Laugt, P. de Mierry, P. Vennegues, H. Lahreche, M. Leroux, and P. Gibart, Appl. Phys. Lett. 79, 3230 (2001).

${ }^{14} \mathrm{~F}$. A. Ponce, in Introduction to Nitride Semiconductor Blue Lasers and Light Emitting Diodes, edited by S. Nakamura and S. F. Chichibu (Taylor \& Francis, London, 2000), Chap. 4, p. 108.

${ }^{15}$ X. J. Ning, F. R. Chien, P. Pirouz, J. W. Yang, and M. Asif Khan, J. Mater. Res. 11, 580 (1996).

${ }^{16}$ A. Krost, J. Blasing, F. Schulze, O. Schon, A. Alam, and M. Heuken, J. Cryst. Growth 221, 251 (2000).

${ }^{17}$ L. B. Rowland, K. Doverspike, and D. K. Gaskill, Appl. Phys. Lett. 66, 1495 (1995). 